
on Metabolomics

\title{
Effects of Algae Feeding on Mouse Metabolome
}

\author{
Yiwei Ma ${ }^{1}$, Wenguang Zhou ${ }^{2}$, Paul Chen ${ }^{2}$, Pedro E. Urriola ${ }^{3}$, Gerald C. \\ Shurson ${ }^{3}$, Roger Ruan $^{2^{*}}$, Chi Chen ${ }^{1^{*}}$
}

${ }^{1}$ Department of Food Science and Nutrition.

2 Department of Bioproducts and Biosystems Engineering.

${ }^{3}$ Department of Animal Science, University of Minnesota, 1334 Eckles Avenue, 225 FScN, St. Paul, MN 55108.

* Corresponding author: chichen@umn.edu

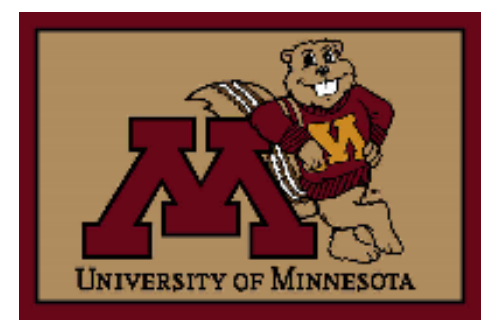


Abstract: The diverse and abundant chemical and nutrient composition in algae makes algae as a source of food, dietary supplement and biofuel. However, the metabolic events in algaeelicited effects were not examined in details. Liquid chromatography-mass spectrometry(LCMS) based metabolomics can help to develop the relationship on the metabolic interactions between algal components and the biological system. Therefore, the influences of consuming different doses of green algae (Scenedesmus sp.) on the metabolic status of young mice was conducted by LC-MS in this study, together with growth performance and blood chemistry. Results from blood chemistry only showed serum cholesterol and TAG was significantly decreased by $20 \%$ algae feeding, while metabolomic analysis of urine, feces, serum and liver samples indicated that algae feeding greatly affected the metabolites belonging to antioxidant, lipid, microbial metabolism and intermediates metabolites in nutrient and energy metabolism. Increased levels of hepatic reduced glutathione, nicotinamide and adenylosuccinate suggested that $5 \%$ algae feeding may upregulate antioxidant system, and increase energy production, which contribute to the growth promotion. In the contrast, the growth suppression effects of $20 \%$ algae feeding was correlated to the increased level of oxidized glutathione and carnitine in the liver, altered lipids profile in serum and liver, and increased acyl-glycine in the urine. Overall, multiple correlations between metabolite markers and growth performance in algae feeding were established in this study and could serve as a foundation for further mechanistic investigations on the biological effects of algae feeding.

Keywords: Microalgae, exposure markers, redox balance, lipidomics, microbial metabolism. 


\section{Introduction}

- Algae are a large and diverse group of polyphyletic and mostly photosynthetic organisms.

- Algae classification:

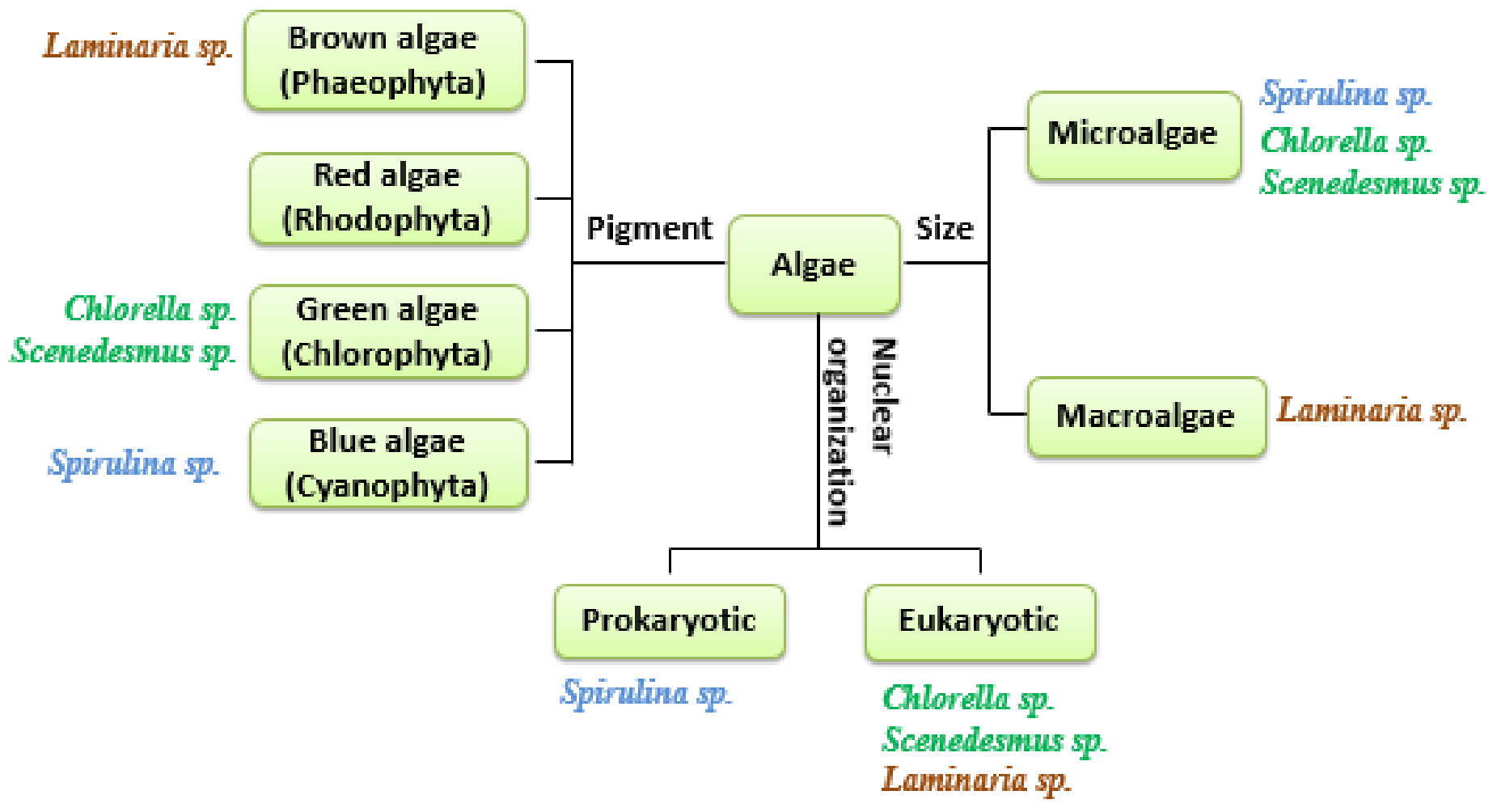




\section{Introduction}

- Application of algae

\section{Human and animal}

\section{Industry}

\section{Environment}

- Dietary supplement

- Functional food

- Feed

- Biofuel

- Pharmaceuticals

- Wastewater treatment 


\section{Introduction}

\section{Macronutrients in algae}

- Carbohydrates: Diverse dietary fiber polysaccharides.

- Lipids: High polyunsaturated fatty acids content. Algal-PUFAs can be incorporated into human foods chain through seafood consumption.

- Protein: High abundance, enriched with certain essential amino acids.

\section{Micronutrients in algae}

- Vitamins: High in water soluble vitamins (B1, B2, B12, C) and fat soluble vitamins (A, E, K).

- Minerals: Good source of potassium, phosphorus, calcium, magnesium and selenium. However, heavy metal contamination, including lead and cadmium, is a concern in algae consumption. 


\section{Introduction}

- Chemical composition of algae is based on their strains, as well as environment parameters such as temperature, $\mathrm{pH}$, illumination, and mineral content.

\begin{tabular}{lcccc}
\hline & Carbohydrate & \multicolumn{1}{c}{ Lipid } & Protein & Nucleic acid \\
\hline Spirulina maxima & $13-16$ & $6-7$ & $60-71$ & $3-4.5$ \\
Spirulina platensis & $8-14$ & $4-9$ & $46-63$ & $2-5$ \\
Chlorella vulgaris & $12-17$ & $14-22$ & $51-58$ & $4-5$ \\
Scenedesmus obliquus & $10-17$ & $12-14$ & $50-56$ & $3-6$ \\
Dunaliella salina & 32 & 6 & 57 & \\
Synechococcus sp. & 15 & 11 & 63 & \\
Cow milk & 38 & 28 & 34 & 43 \\
Meat muscle & 1 & 20 & 37 & \\
Soybean & 30 & &
\end{tabular}

*Value showed in \% of dry matter Data from E.W. Becker, Biotechnology and microbiology. 1994. Cambridge Press. 


\section{Introduction}

- Scenedesmus sp. is selected due to its high eicosapentaenoic acid (C20:5) content.

(\% of total fatty acids)

\begin{tabular}{lccccc}
\hline & $\begin{array}{c}\text { Spirulina } \\
\text { maxima }\end{array}$ & $\begin{array}{c}\text { Spirulina } \\
\text { platesis }\end{array}$ & $\begin{array}{c}\text { Chlorella } \\
\text { pyrenoidosa }\end{array}$ & $\begin{array}{c}\text { Chlorella } \\
\text { vulgaris }\end{array}$ & $\begin{array}{c}\text { Scenedesmus } \\
\text { sp. }\end{array}$ \\
\hline C12:0 & 0.52 & 0.84 & 1.07 & 0.38 & n.d. \\
C14:0 & n.d. & n.d. & 0.58 & 0.69 & $\mathbf{4 . 1 2}$ \\
C16:0 & 35.82 & 42.30 & 14.60 & 15.41 & $\mathbf{2 6 . 9 2}$ \\
C16:1 & 0.85 & 1.00 & 3.07 & 1.17 & 5.77 \\
C16:2 & 4.61 & 2.43 & 5.10 & n.d. & $\mathbf{2 . 7 5}$ \\
C16:3 & n.d. & n.d. & 3.16 & n.d. & n.d. \\
C18:0 & 1.49 & 0.95 & 1.93 & 6.24 & $\mathbf{0 . 8 7}$ \\
C18:1 & 5.03 & 1.97 & 18.24 & 33.14 & $\mathbf{3 1 . 7 3}$ \\
C18:2 & 16.34 & 16.18 & 11.24 & 9.73 & $\mathbf{1 5 . 8 6}$ \\
C18:3 (w-3) & N/A & N/A & 15.87 & 1.93 & $\mathbf{0 . 5 6}$ \\
C18:3 (w-6) & 18.16 & 20.06 & N/A & N/A & N/A \\
C20:0 & n.d. & n.d. & n.d. & 0.19 & $\mathbf{0 . 1 4}$ \\
C20:1 & n.d. & n.d. & n.d. & n.d. & $\mathbf{0 . 1 3}$ \\
C20:2 & 0.59 & 0.96 & 0.71 & n.d. & n.d. \\
C20:5 & n.d. & n.d. & 0.40 & 3.23 & $\mathbf{7 . 5 8}$ \\
Saturated & 46.31 & 51.64 & 33.00 & 22.22 & $\mathbf{3 5 . 6 2}$ \\
Monounsaturated & 11.24 & 5.88 & 23.98 & 35.44 & $\mathbf{3 7 . 6 2}$ \\
polyunsaturated & 40.36 & 39.51 & 36.48 & 38.94 & $\mathbf{2 6 . 7 6}$ \\
\hline
\end{tabular}




\section{Introduction}

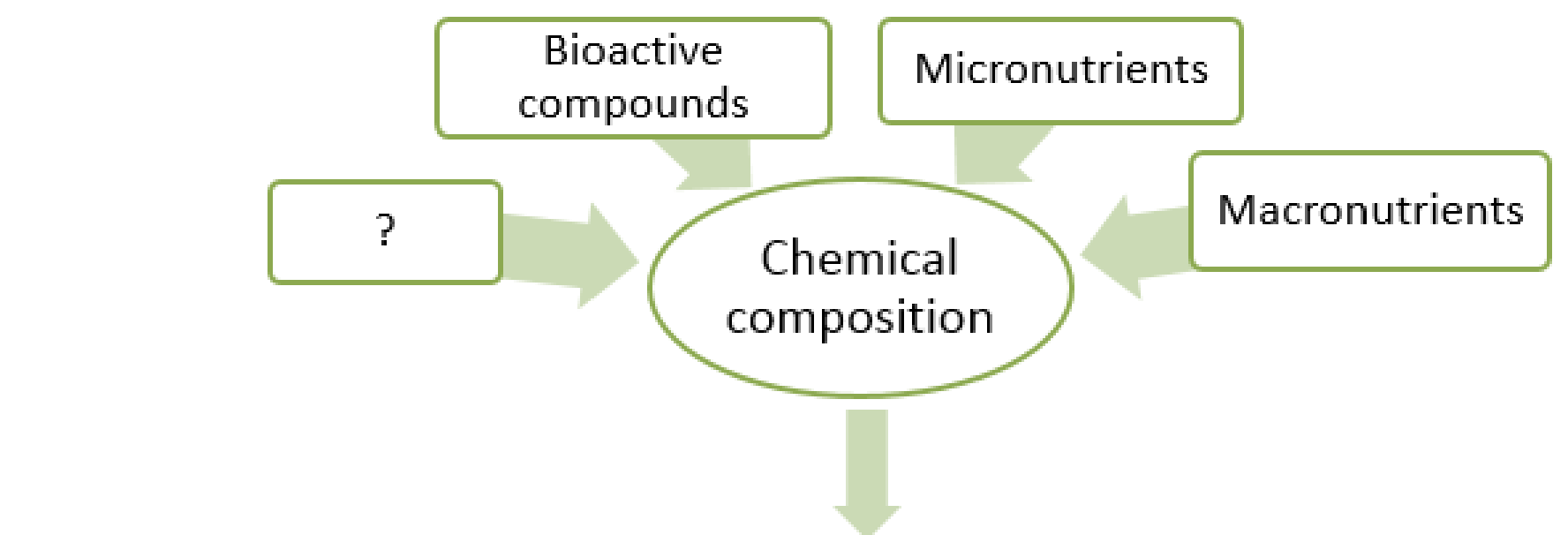

Metabolomics

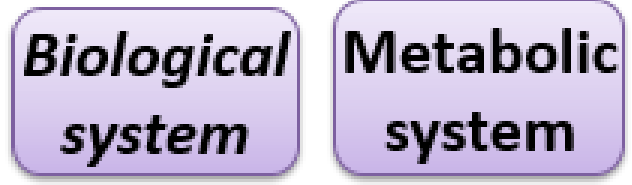

Algae-induced

biological

effects 


\section{Introduction}

- Metabolomics is the systematic study of metabolites.

\section{Genes}

$$
\text { mRNA }
$$

Proteins

Metabolites

Disease

Diet

- Compared to other "-omics", metabolomic analysis can reveal the information more closely related to the physiology of a biological system.

- Therefore, metabolomics could improve our understanding on how algae influence the phenotype. 


\section{Objectives}

- To investigate the influences of feeding Scenedesmus algae on mouse metabolome.

- To correlate the metabolic effects of algae feeding with growth performance and health status. 


\section{Experiment design}

- Animals:

C57BL/6 mice, 8 weeks old

- Treatments:

Control AIN93G $(n=8) ; 5 \%$ algae $(n=8) ; 20 \%$ algae $(n=8)$

- Body weight and food intake were measured every other days

- Urine, feces, serum, and tissue samples were collected at the end of 4-week feeding for blood chemistry analysis and LC-MS-based metabolomics.

- Statistic significance was evaluated by one-way ANOVA using the PROC GLM and Tukey - Kramer comparison test using the PROC GLM procedure of SAS .

- Data are shown as the means \pm SD 


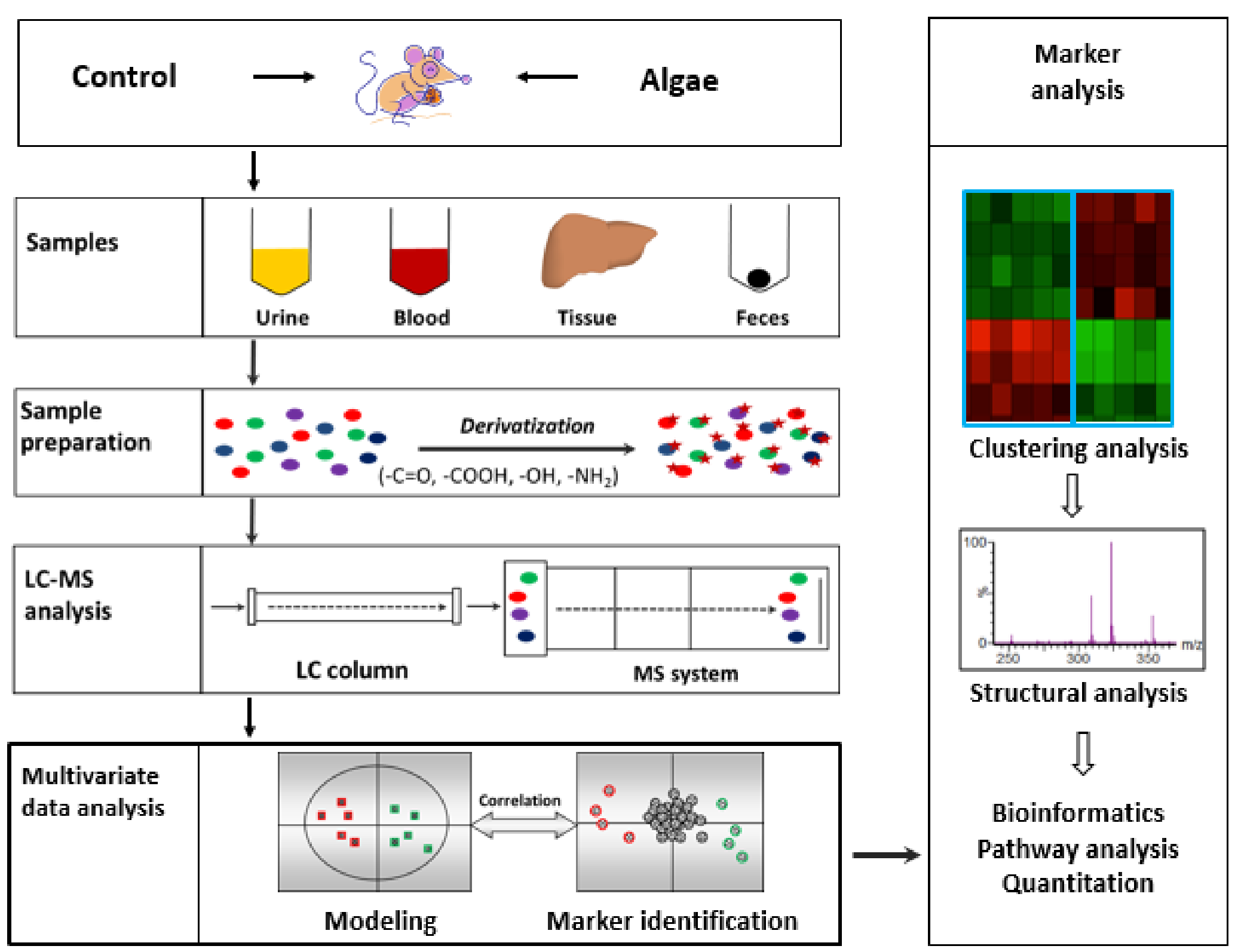

2nd International Electronic Conference on Metabolomics

20-27 November 2017 


\section{Metabolomics: sample preparation}

* Fractionation: to separate the aqueous and lipid fractions from the liver and serum.

* Chemical derivatization: for separation and detection

---Dansyl chloride for amines and alcohols.

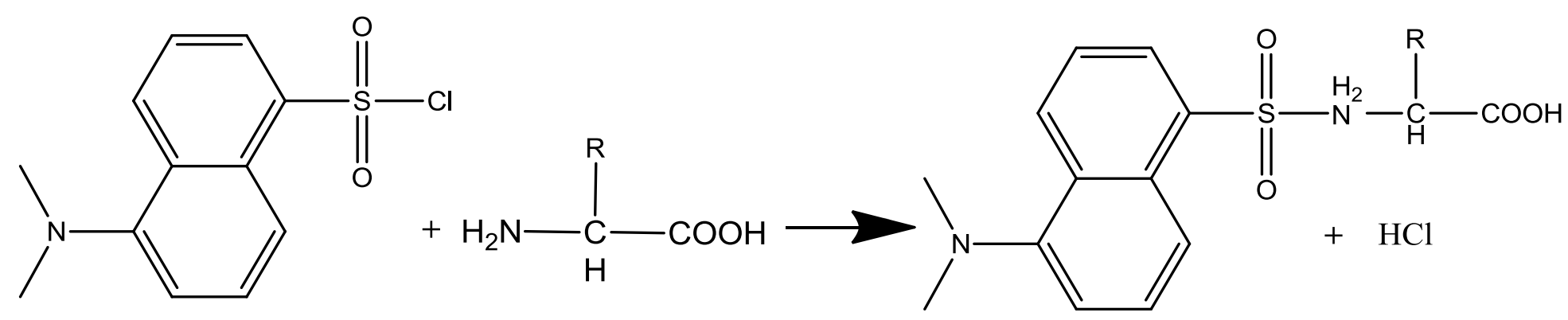

---2-Hydrazinoquinoline (HQ) for carboxylic acids, ketone and aldehydes.

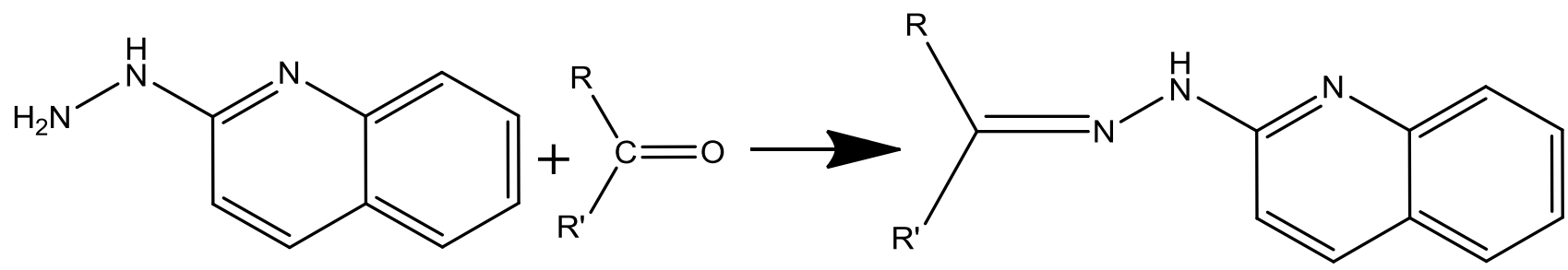




\section{Metabolomics: multivariate data analysis (MDA)}

- Chromatographic and spectral data of urine, feces, serum and liver samples were deconvoluted by MarkerLynx software (Waters).

- Data extracted from the chromatograms and mass spectra of urine, feces, serum and liver samples were processed by a partial least-squaresdiscriminant analysis (PLS-DA), a supervised MDA method in SIMCA-P+ software. A two-component model was further constructed to delineate the relationship among sample groups as well as the contribution of eat detected chemical ion to the principal components (PCS) of the multivariate model.

- The $t[1]$ and $t$ [2] values in the scores plot represent the scores of each sample in the principal components 1 and 2, respectively. The model was validated through the recalculation of $R^{2}$ and $Q^{2}$ values after the permutation of sample identities. 


\section{Metabolomics: marker identification}

- Accurate mass-based database search: HMDB, KEGG, LipidMap

- Confirmation with authentic standards

- MS/MS fragmentation for structural elucidation 


\section{Results}

\section{General responses to algae feeding}
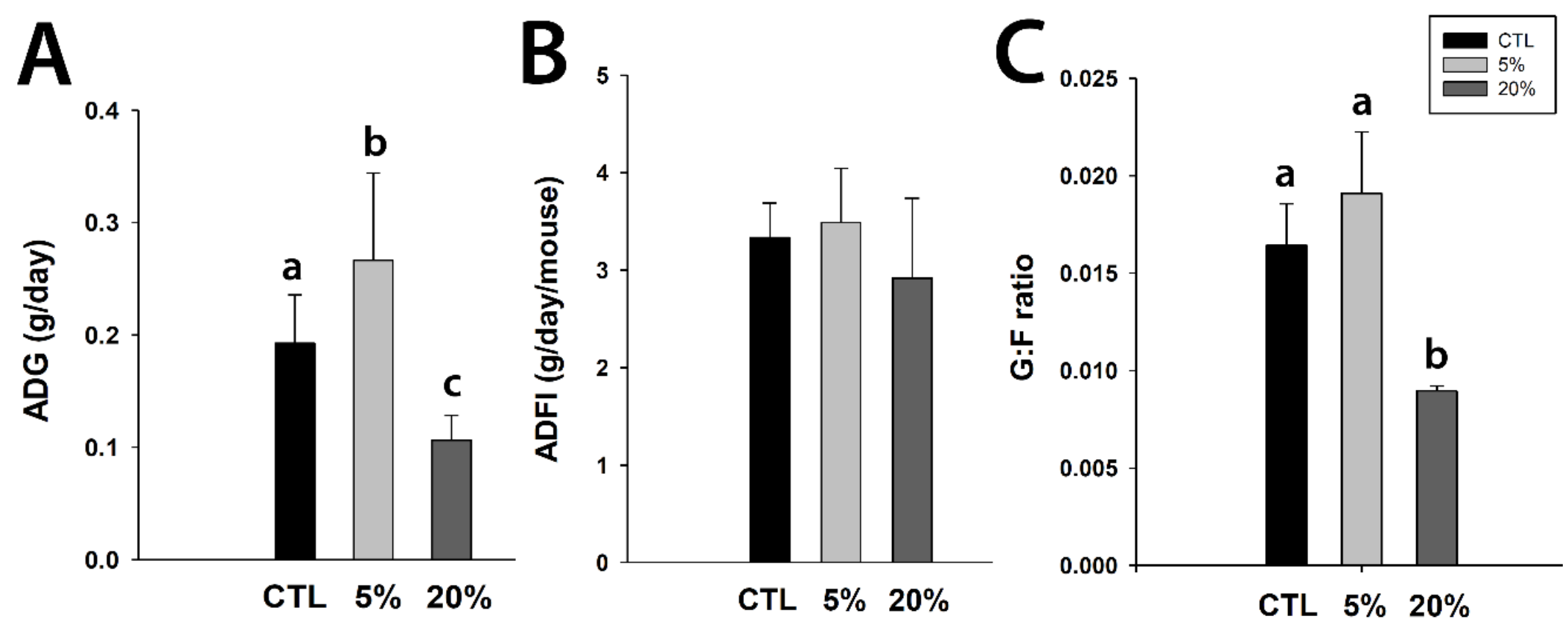

- $5 \%$ algae feeding promoted the growth while $20 \%$ suppressed it. 


\section{Results}

\section{Effects on blood chemistry}
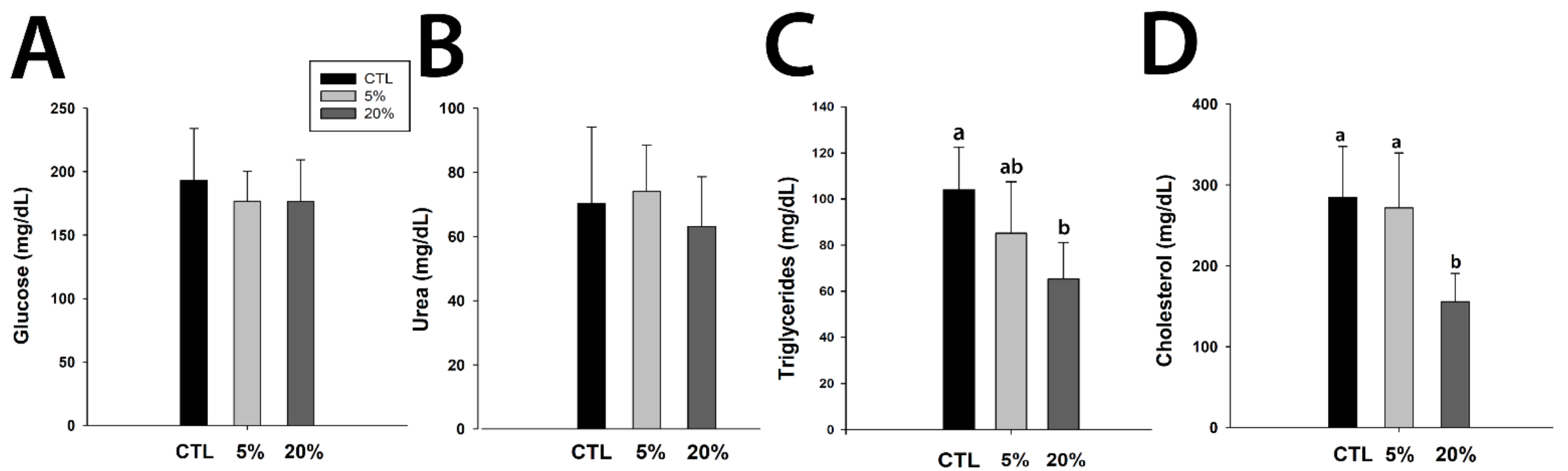

- Serum glucose, BUN, ALT, and AST were not affect by algae treatments, but TAG and cholesterol was significantly decreased by $20 \%$ algae feeding. 


\section{Results --- Effects on metabolomes}

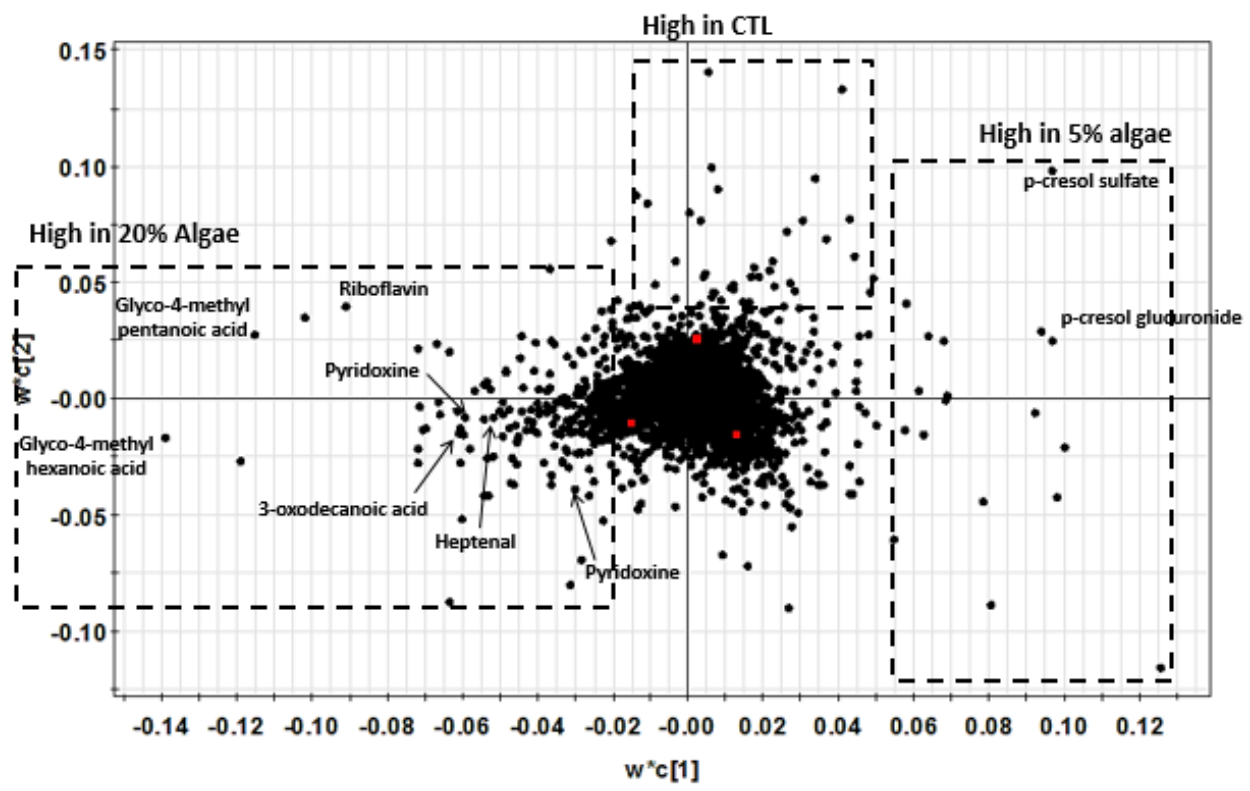

Urine sample

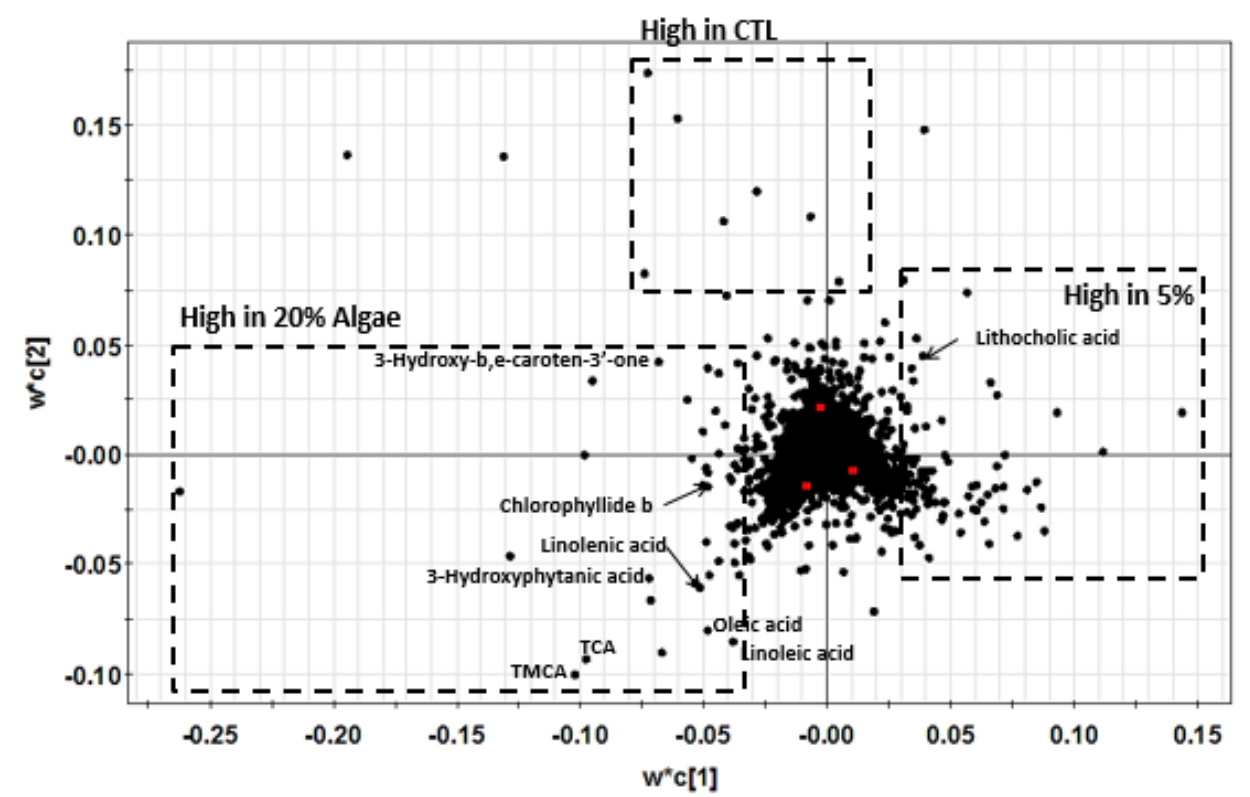

Feces sample
Comp No. M2.R2Y(cum) M2.Q2(cum)

\begin{tabular}{lll}
\hline Comp[1] & 0.476045 & 0.422003 \\
Comp[2] & 0.879791 & 0.625485 \\
\hline
\end{tabular}

Comp No. M2.R2Y(cum) M2.Q2(cum)

\begin{tabular}{lrr}
\hline Comp[1] & 0.49194 & 0.483151 \\
Comp[2] & 0.954157 & 0.919335 \\
\hline
\end{tabular}

2nd International Electronic Conference on Metabolomics

20-27 November 2017 


\section{Results --- Effects on metabolomes}

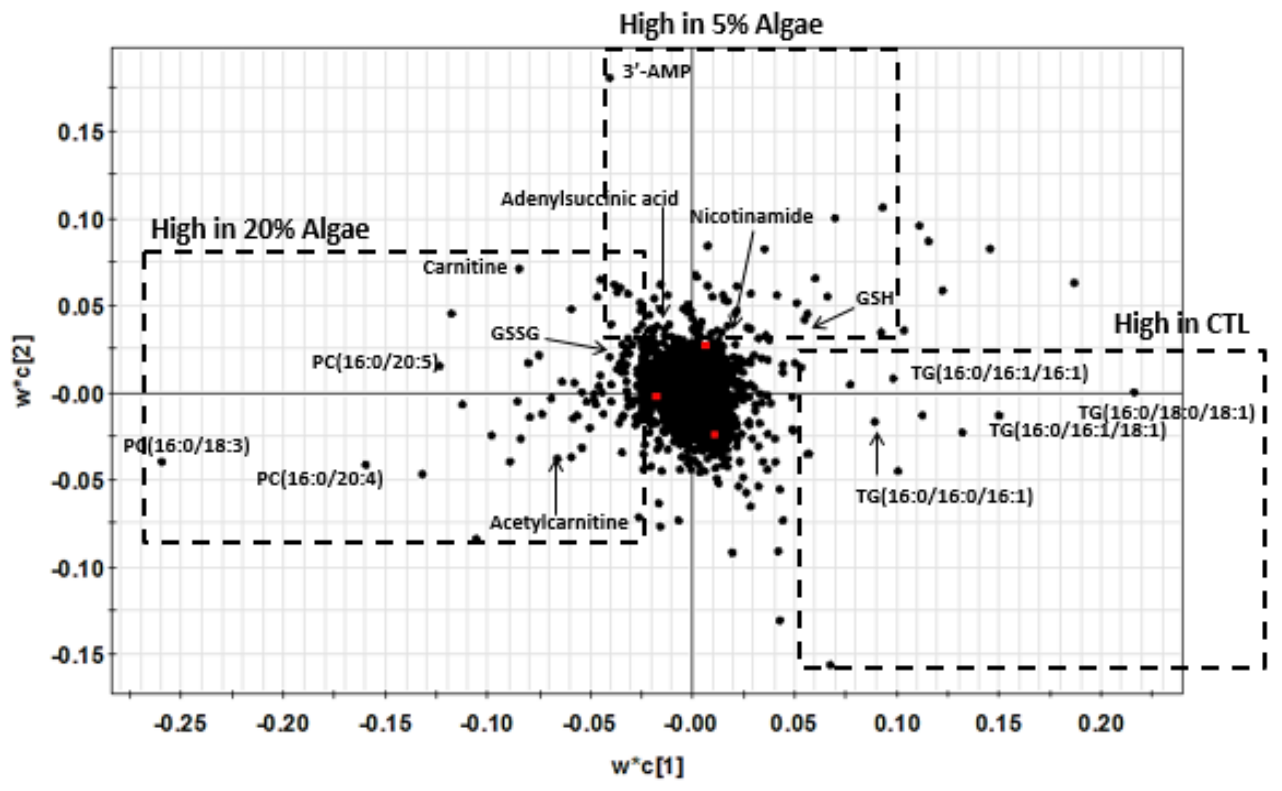

Liver sample

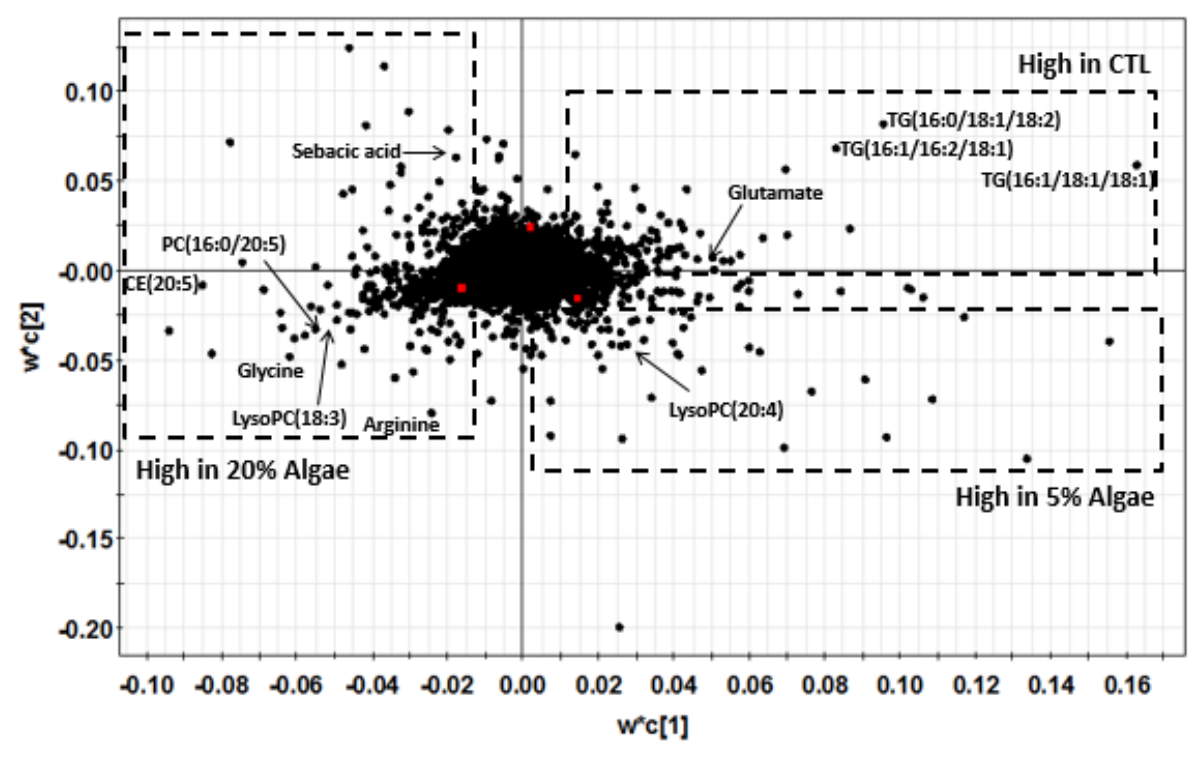

Serum sample

\begin{tabular}{lrr} 
Comp No. & M2.R2Y(cum) & M2.Q2(cum) \\
\hline Comp[1] & 0.454237 & 0.403972 \\
Comp[2] & 0.87008 & 0.630112 \\
\hline
\end{tabular}

Comp No. M2.R2Y(cum) M2.Q2(cum)

\begin{tabular}{lll}
\hline Comp[1] & 0.466327 & 0.378317 \\
Comp[2] & 0.897856 & 0.665836 \\
\hline
\end{tabular}




\section{Results}

Algae chemicals

\section{Endogenous metabolism}

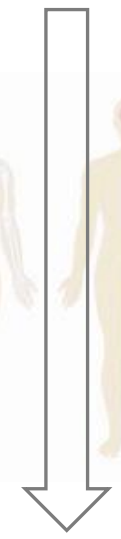

Algae specific compounds as exposure markers
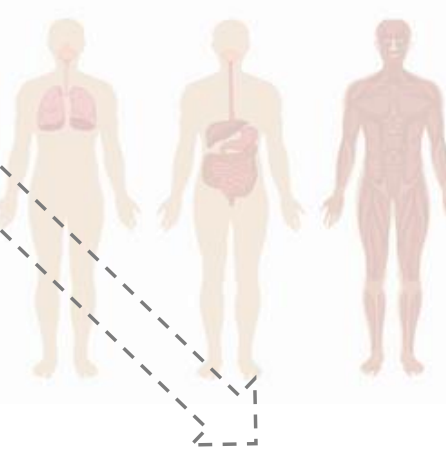

Macronutrient metabolites
Intermediate metabolites, microbial metabolites 


\section{Results}

Exposure markers

- Pantothenic acid was only increased by $20 \%$ algae feeding.

- Pyridoxine and riboflavin were increased by algae dose-dependently.

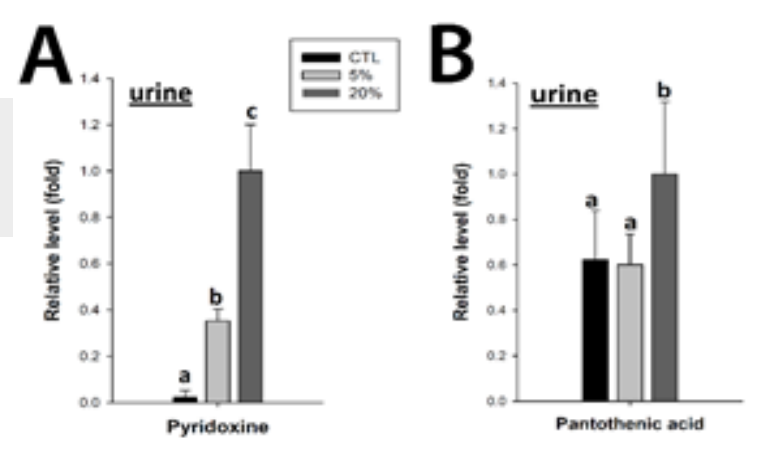

$\mathbf{E}$

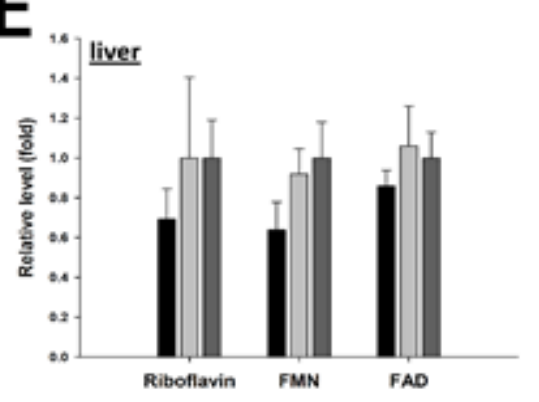

$\mathbf{F}$
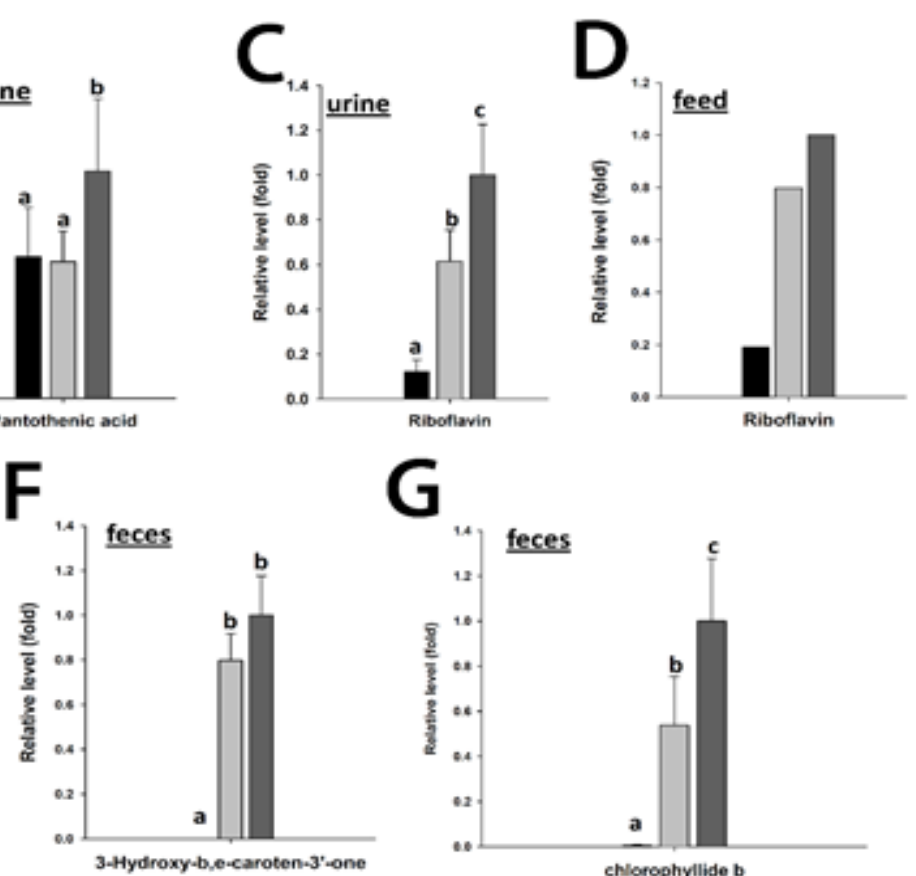

G

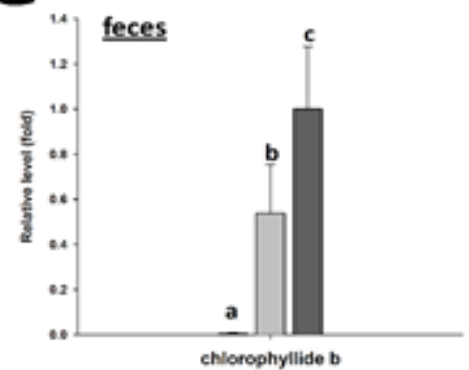

- Extra riboflavin from algae might not been effectively retained inside the body.

- 3-hydroxy-b,e-caroten-3'one is a degradation product of carotenoids.

- Chlorophyllide b is a component of algal chloroplast. 


\section{Results}

Macronutrients-(amino acids)
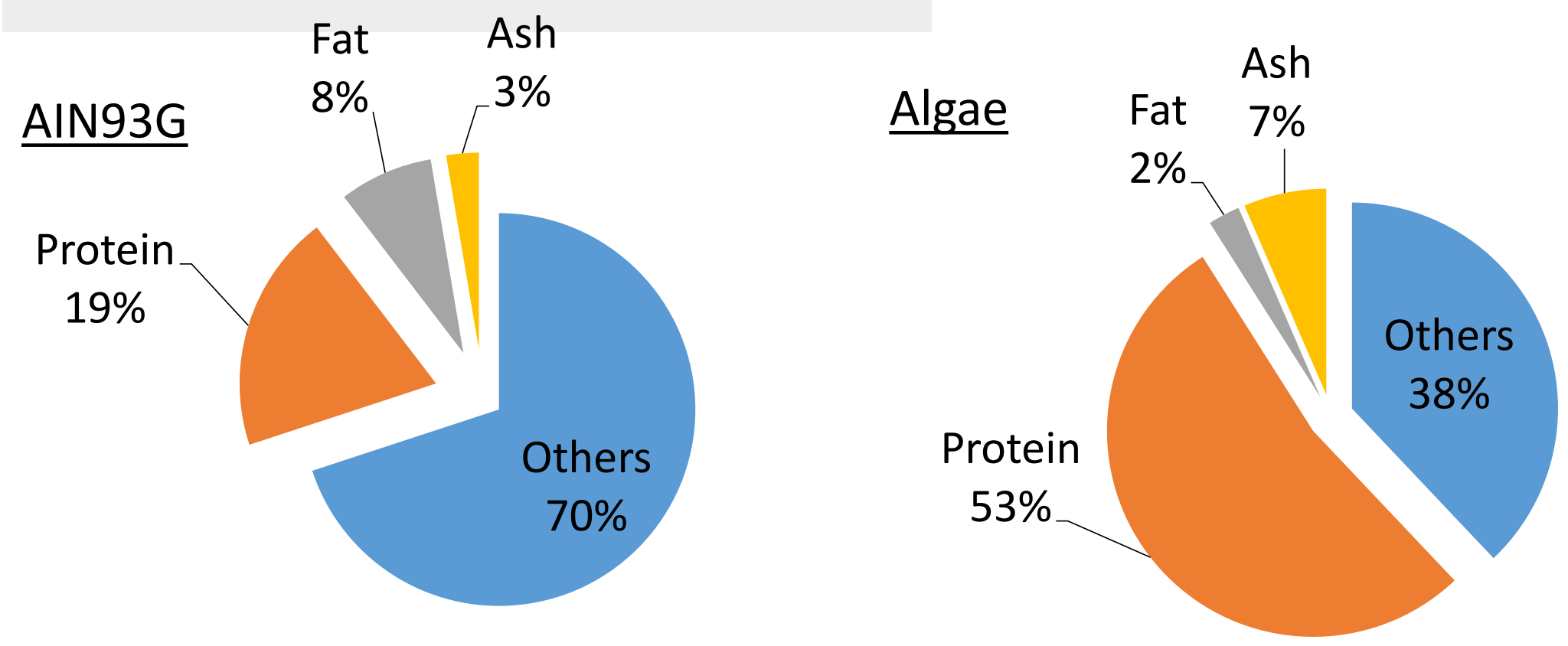

- In both liver and serum, taurine was decreased, while arginine and citrulline were increased by $20 \%$ algae feeding.

- In serum alone, algae feeding was associated with the changes in methionine, glycine, lysine, aspartate and threonine. 


\section{Results}

\section{Macronutrients} -(lipids)
HCA-based heat map on liver lipid markers
$\mathrm{PC}(16: 0 / 20: 5)^{*}$ $\mathrm{PE}(18: 0 / 22: 6)^{\star}$ $\mathrm{PC}(16: 0 / 16: 0)^{\star}$ TG(18:2/18:2/22:6) * TG(18:2/18:1/22:6)* Lyso(16:0) $\mathrm{PC}(16: 1 / 18: 3)^{*}$ $\mathrm{TG}(18: 1 / 18: 1 / 22: 5)^{\star}$ $\mathrm{PE}(18: 0 / 20: 4)^{*}$ $\mathrm{PC}(16: 0 / 20: 4)^{*}$ $\operatorname{PE}(18: 1 / 20: 4)^{*}$ $\mathrm{PC}(18: 0 / 18: 2)^{*}$ $\mathrm{PC}(16: 0 / 18: 3)^{\star}$ $\mathrm{PC}(18: 1 / 22: 6)^{*}$ $\mathrm{PE}(16: 0 / 22: 6)^{*}$ $\mathrm{PC}(18: 0 / 22: 6)^{*}$ LysoPC(18:2) TG(16:0/18:2/20:5) TG(16:0/16:1/20:5) $\mathrm{TG}(18: 1 / 18: 1 / 22: 6)^{*}$ $\mathrm{TG}(18: 2 / 16: 0 / 22: 6)$ TG(16:0/16:1/16:1) TG(18:1/18:1/20:1) TG(16:0/16:0/16:1) TG(16:1/16:0/18:1)* $\mathrm{TG}(16: 0 / 18: 1 / 18: 1)^{*}$ TG(18:0/18:1/18:1) TG(16:0/18:1/18:2)* TG(16:1/16:1/18:1) TG(16:0/18:0/20:4) TG(18:2/18:2/20:2) TG(16:1/16:1/18:2) TG(16:0/18:1/20:4) LysoPC(20:4) TG(18:1/18:1/20:5)
HCA-based heat map on serum lipid markers

M(d18:0/16:1)

TG(20:5/18:2/22:6) G(20:4/18:1/20:5 TG(20:5/18:1/22:6) CE20:5

PC(18:0/18:2) PE(16:0/22:6) PC (16:0/20:5) PC 18:0/20:2 PC $18: 3 / 22: 6$ PC (15:0/18:2) PE (16:0/20:0) PC (20:0/22:6) PC(16:0/18:2) LysoPC (20:5) C(20:0/20:4) TG(18:2/18:2/18:3) TG(18:2/18:2/20:4) PC(16:0/22:6)* PC (18:1/20:4) PS 19:0/20:5

T. TG(16:0/18:1/18:2) TG(16:1/16:2/18:1) LysoPC(20:4)*

TG(18:1/18:1/18:2)* LysoPC(22:6)*

ysoPC(20:3)*

TG(16:0/18:1/20:1) LysoPC (18:1)

TG(18:1/18:1/18:1) TG(16:1/18:1/18:1) TG(16:0/16:0/18:1

PC(16:1/0:0)

LysoPC (20:0) LysoPC 18:2

TG(18:1/18:1/20:3)

LysoPC(18:0)*

TG(18:1/18:2/20:1)*

LysoPC $(16: 0)$ PC(18:0/20:3)* PC(16:0/22:4) PC (20:4/22:6)* PC 18:0/22:6)* TG(18:1/18:1/20:1)* $\mathrm{PC}(18: 1 / 20: 3)^{*}$ 


\section{Results}

Intermediate metabolites

- GSH was positively correlated with $5 \%$ algae feeding, while GSSG with $20 \%$.

- GSH/GSSG ratio was slightly increased.
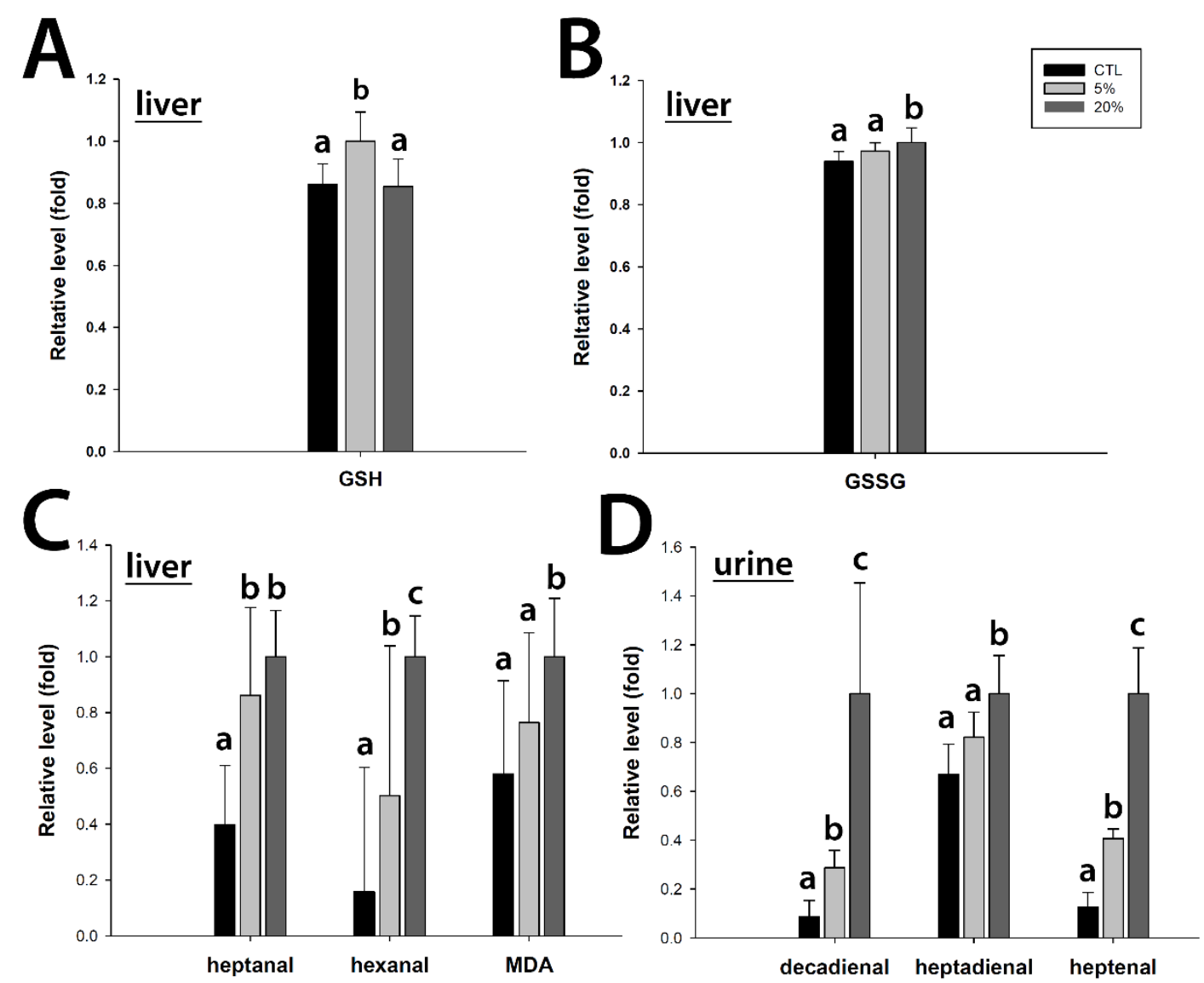

- $20 \%$ algae feeding elicited a higher level of oxidative stress while $5 \%$ algae feeding was associated with the upregulation of antioxidant system. 


\section{Results}

\section{Microbial metabolites}

- Short chain fatty acids in feces were significantly increased by both algae feeding, except for acetic acid.

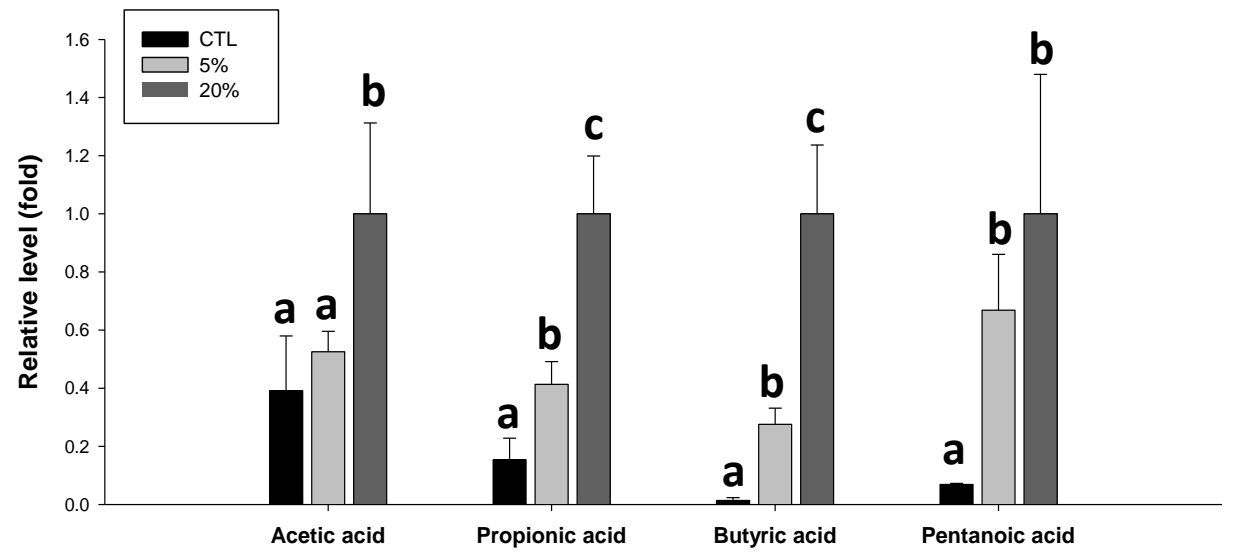

- Primary bile acid (MCA) was increased, while secondary bile acid (LCA) was decreased.

- Taurine bile salts were dramatically and dose-dependently increased by both algae feeding.

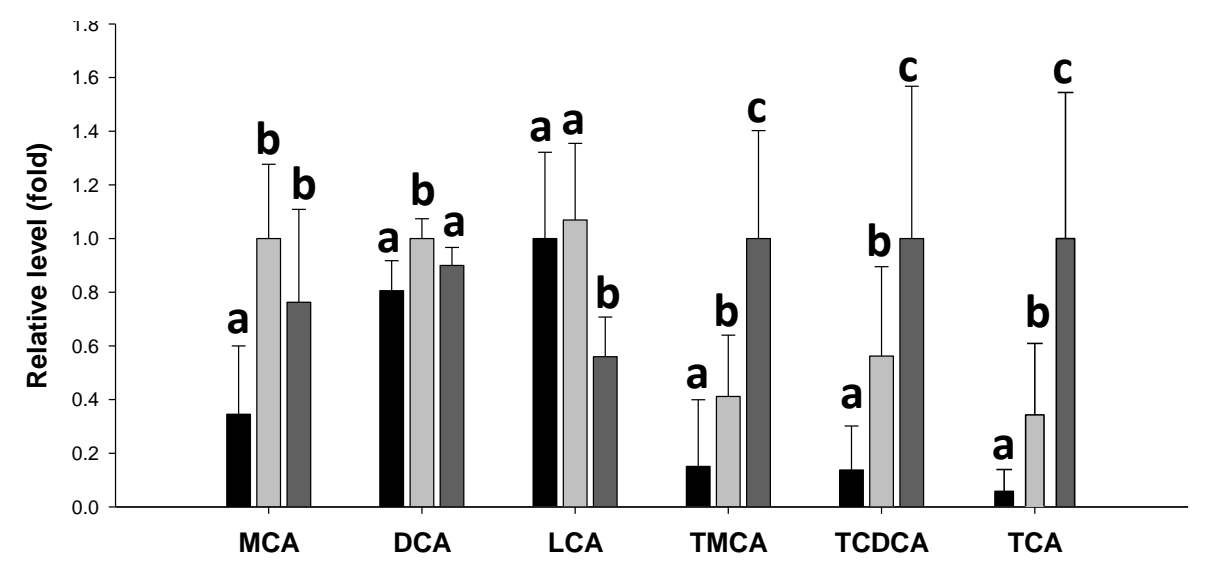

- In urine, $\mathrm{p}$-cresol metabolites derived for microbial degradation of tyrosine were decreased by $20 \%$ algae feeding.

- BCFAs from bacterial metabolism were increased by $20 \%$ algae feeding.
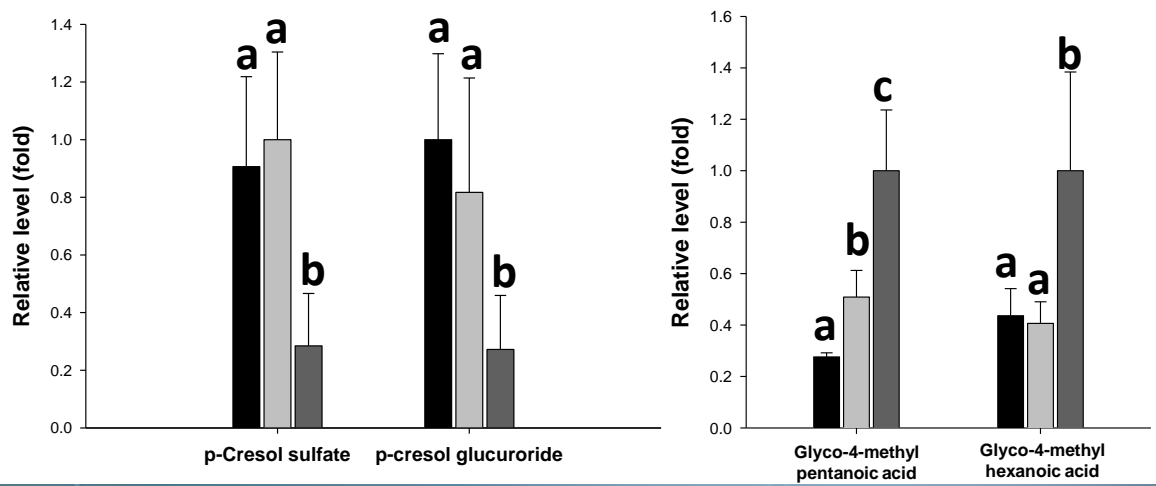


\section{Discussions}

- Compared to limited coverage of blood chemistry analysis, metabolomics provides comprehensive coverage on algae-induced metabolic changes.

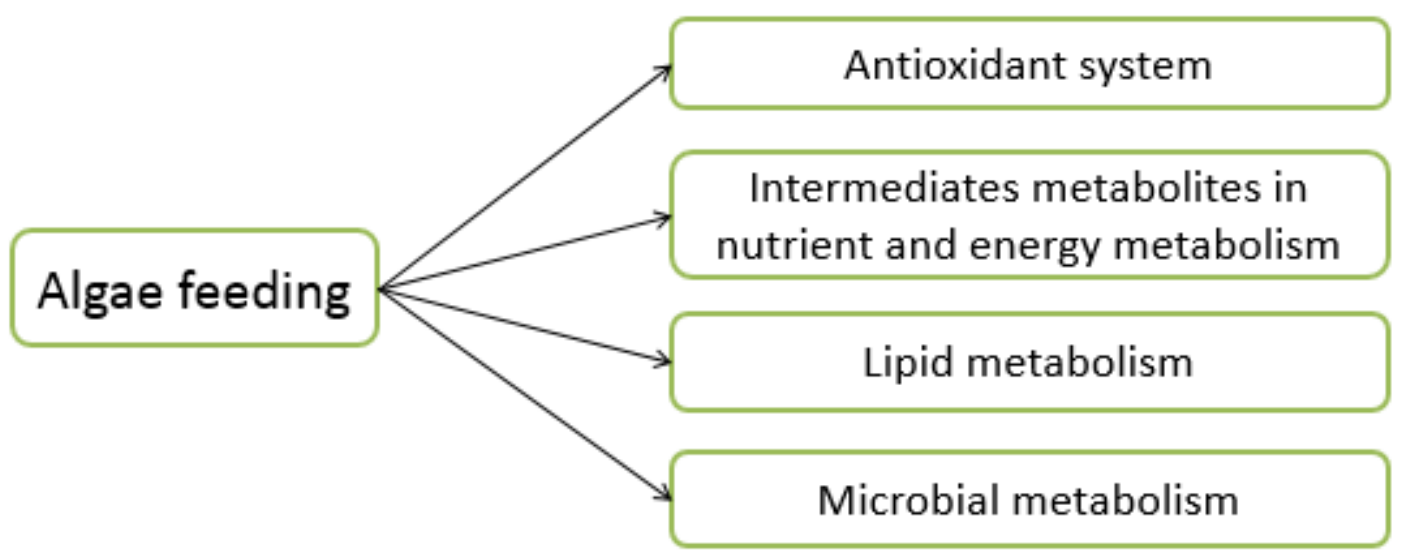

- $5 \%$ algae feeding had growth-promoting effect, while $20 \%$ algae feeding had hypolipidemic and growth-suppressing effect.

- The increases in B vitamins and PUFA were observed after algae consumption, but not positively correlated with growth performance, especially after $20 \%$ algae feeding. 


\section{Discussions --- marker of $5 \%$ algae feeding}

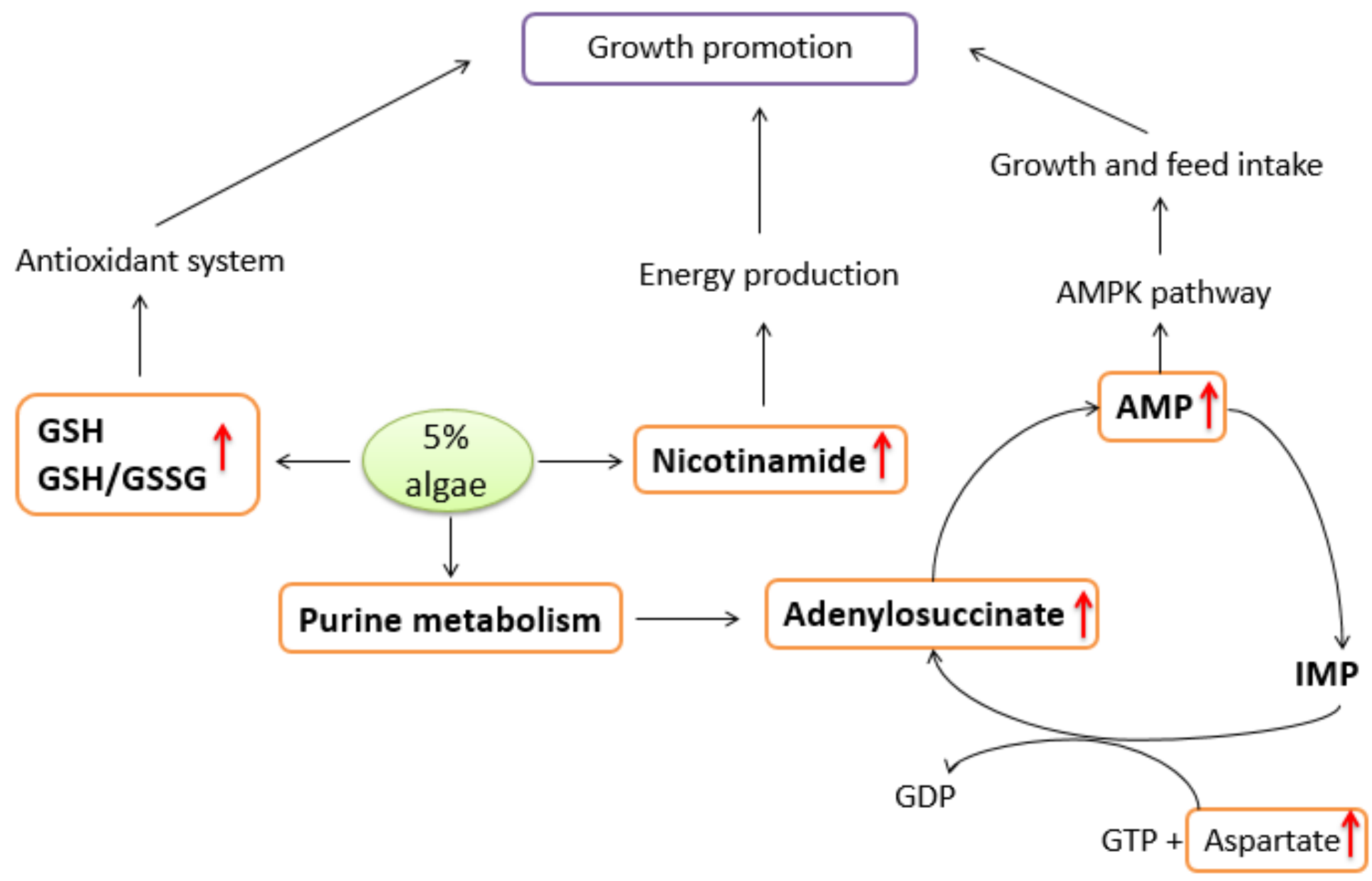




\section{Discussions --- marker of $20 \%$ algae feeding}

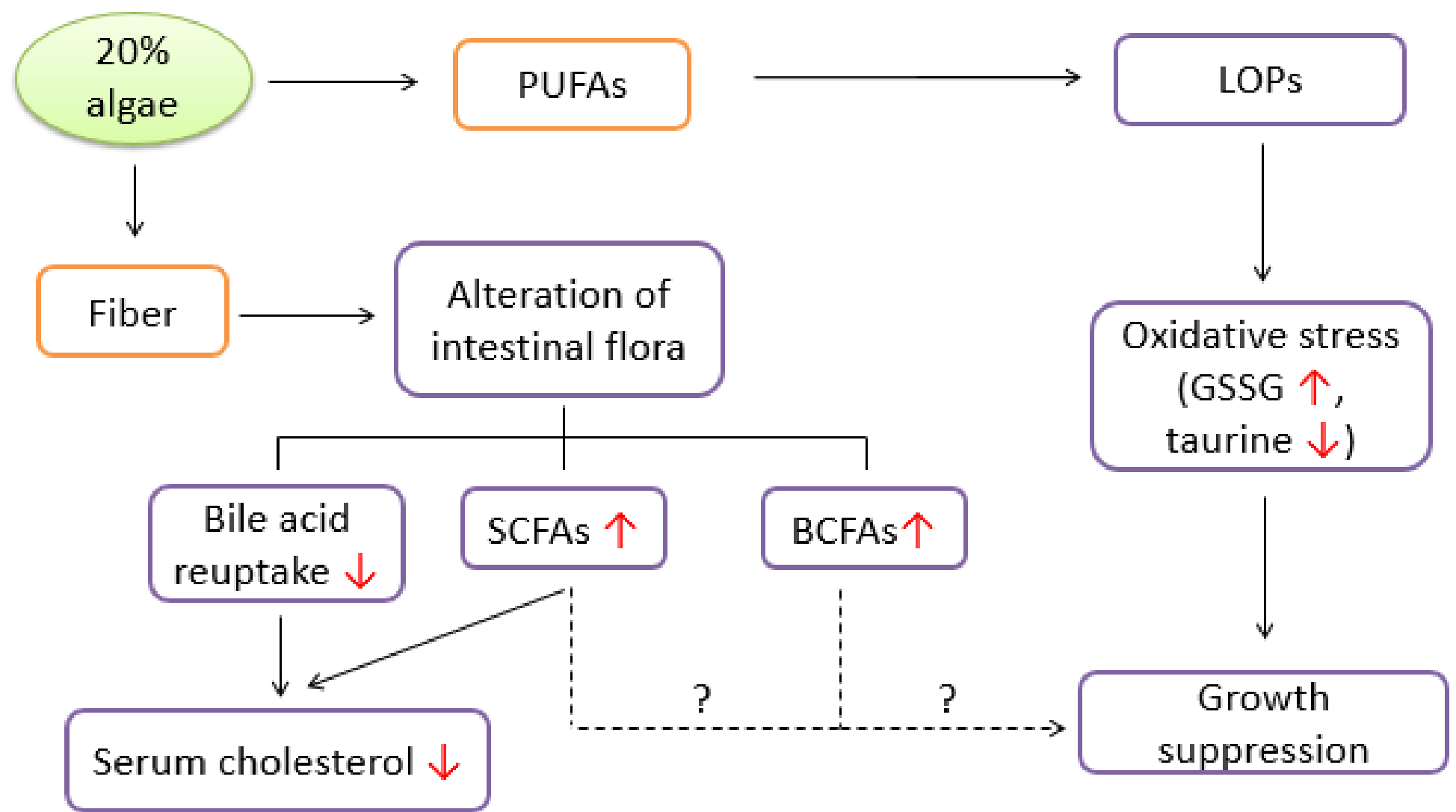




\section{Conclusions}

- The dose-dependent correlations between algae feeding and growth performance observed in this study, together with the observations for other feeding experiments in mice and pigs, suggest that there might be a threshold dose in algae feeding for inducing positive or negative responses. More mechanistic investigations are required to define the metabolic and signaling events behind these observations.

- All the findings can serve as a foundation for establishing biomarkers of algae-induced biological effects and conducting further investigation to guide the uses of algae as dietary supplements in humans and feed in animals. 


\section{Acknowledgments}

- Advisor: Chi Chen

- Co-Advisor: Roger Ruan

- Dr. Roger Ruan's lab: Dr. Joe Zhou, Dr. Paul Chen

- Dr. Chi Chen's lab: Dana Yao, Lei Wang, Feng Ding, Qingqing Mao, John Kurtz, Tina Bi

- This project was supported by the MNDRIVE. 